\title{
Approach in the modeling of wind turbines for power system stability studies and evaluation of their impact on electric power systems
}

\author{
Igor Razzhivin ${ }^{1, *}$, Almaz Sulaimanov $^{1}$, and Sergey Stavitsky ${ }^{1}$ \\ ${ }^{1}$ National Research Tomsk Polytechnic University, 634050 Tomsk, Russia
}

\begin{abstract}
Wind power - one the fastest evolving in renewable energy industry. From year to year the share of wind energy in power systems are increasing as well as increasing the size of wind farms. A large-scale wind farms that produce hundreds of megawatts of electricity have a serious impact on the power grid. This paper discusses proposed approaches to modeling wind farms using the Hybrid Real-Time Power System Simulator (HRTSim), to study stability and transient processes in electric power systems.
\end{abstract}

\section{Introduction}

A renewable energy sources play an increasingly important role in energy supply as a result of the constant increase prices on fuel and environmental problems in world. According to experts for 2020, expectations for renewable energy to $280 \mathrm{GW}$ will be installed in Europe, which is $64 \%$ of its total planned capacity, and the installed capacity of wind farms will be $136 \mathrm{GW}$ (59\%). Large wind farms in Europe, China and the United States consist of hundreds of wind turbines with an installed capacity exceeding 1,000 MW. For example, the wind farm in Gansu Province (China) has more than 5,000 turbines with more than 5 GW a total capacity, and is underway to expand it [1-2].

The growing scale of large wind farms and penetration level of wind power, consider the chaotic nature of its production would consequently exert serious impacts on the operation and control of the electric power systems (EPS), that wind power is integrated. Issues of stability of EPS, regulation of reactive power, voltage, active power, frequency, etc. remain today not fully investigated today are not fully investigated. It is therefore very important to study the interaction between large-scale wind farms and power system connected, and the impacts caused by wind power integration. To fulfil this purpose, adequate wind farm models are required.

Conceptually, there are two approaches to modeling wind farms: detailed modeling and aggregated modeling. In detail, all wind turbines in the wind farm and their interconnection are modeled. For a wind farm with a high number of wind turbines the detailed model is a complex system and leads to a long calculation time of the models in the hardware and software simulation tools. Aggregated modeling uses one or more equivalent wind turbines

\footnotetext{
Corresponding author: lionrash@tpu.ru
} 
and is most often used to study transient processes and the stability of a power plant with a high proportion of wind energy [3].

In this article, it is proposed to apply HRTSim as a tool for modeling wind farms in EPS, taking into account all the advantages of this software-hardware real time system.

\section{Wind farm modeling}

The accuracy of the model of wind turbines has a decisive influence on the results of wind park modeling. Setting the goal of research in studying the complex reaction of wind farms, the impact on power stability when connecting high-power wind farms to the power system, the interaction of the wind farm with other power plants, etc., it is necessary to use accurate and reliable models of all components of the wind farm.

Using a detailed approach in modeling leads to a high complexity of modeling and long calculations and exhausting computation time, especially for wind farms with a large number of wind turbines. Hardware and software simulation tools appropriate for modeling failing with this problem in virtue of the limited mathematical calculations, and the impossibility of solving large systems of differential equations describing processes in EPS. Moreover, when solving the systems of differential equations by numerical methods, the computational error accumulates, which reduces the reliability of the simulation results [46]. Various scientific community and research laboratories when modeling large wind parks are necessity to apply certain limitations and assumptions, applying equivalent models in calculations. Therefore, to solve the above-mentioned problems and obtain the most accurate results in the simulation of wind farms the HRTSim was chosen as the modeling instrument [7-8].

Modeling of wind turbine systems requires interdisciplinary knowledge. The wind turbine has a complex device and combines an aerodynamic model, a mechanical model and an electric model, as well as a control system model. In Fig. 1 shows the general structure of the wind turbine model and the input parameters.

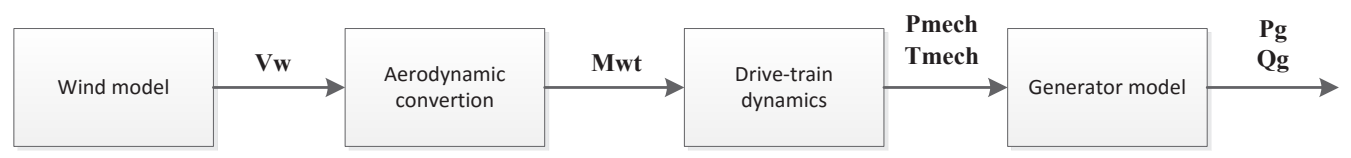

Fig. 1. The basic structure of the overall wind turbine system.

\subsection{Wind model}

In modeling the wind usually take a constant value equal to the average wind speed or wind define the mathematical model through a sine function which is not quite correct assumption in large wind farms assess the impact on the EPS.

To simulate the dynamic component of wind speed in the HRTSim there is provided to implement the spectral model of Kaimal and Davenport recommended by the international standard [9-11].

$$
V(t)=\bar{V}+\Delta V(t)
$$

where: $\bar{V}$ is the average wind speed; $\Delta V(t)$ - deviation of wind speed (dynamic component of speed)

The average wind speed is given as a function of time. The statistical characteristics of the dynamic component $\Delta V(t)$ are specified using the spectral density function $S(f)$.

To calculate the dynamic component of wind speed, the spectral density model of Kaimal is described: 


$$
\frac{f \cdot S(f)}{\sigma^{2}}=\frac{4 \cdot f \cdot L / \bar{V}}{(1+6 \cdot f \cdot L / \bar{V}}
$$

where: $f$ - the frequency of the harmonic component of the wind speed signal, Hz; $S(f)$ one-sided spectrum of horizontal component of wind speed; $\sigma$ - root-mean-square deviation of the horizontal component of wind speed; $L$ - the turbulence coefficient.

Using Fourier Theorem function of the horizontal component of the wind speed may be represented as a series:

$$
V(t)=A_{0}+\sum_{k=1}^{\infty} A_{k} \cdot \operatorname{Cos}\left(\omega_{k} t+\varphi_{k}\right),
$$

where $A k$ - the amplitude of the k-th wind speed harmonics; $\omega_{k}$ - the circular frequency of the corresponding harmonic; $\varphi_{k}$ - the phase shift of the $k$-th vibration; $A_{0}$ - the probability.

Then the amplitude of the k-th harmonic is equal to:

$$
A_{k}=\sqrt{S\left(f_{k}\right) \Delta f}
$$

where $\Delta f$ - the interval between neighboring frequencies.

Substituting the corresponding values, we get that the horizontal wind speed in the time interval $\mathrm{T}$ is equal to:

$$
V(t)=\bar{V}+\sum_{k=1}^{N} A_{k} \cdot \operatorname{Cos}\left(\omega_{k} t+\varphi_{k}\right)
$$

where $\bar{V}$ is the wind speed averaged over a given time interval.

To implement the aerodynamic model of the turbine A mathematical model is proposed that allows to reproduce the torque of the wind turbine $\mathrm{M}_{\mathrm{wt}}$ in accordance with the equation used in their design and reliably verified practice of its application:

$$
\mathrm{M}_{\mathrm{wt}}=\mathrm{M} \pi \mathrm{R}^{3} \frac{\mathrm{pv}^{2}}{2},
$$

in which $\mathrm{R}$ is the radius of the wind wheel, $\mathrm{V}$ is the wind speed, and $\mathrm{M}$ is determined by the structural features and parameters of the wind turbine wheel:

$$
\mathrm{M}=\frac{4 \mathrm{e}}{\mathrm{Z}} \cdot \frac{1-\mathrm{e}}{1+\mathrm{e}}\left[\left(1-\frac{\mathrm{r}_{0}^{2}}{\mathrm{R}^{2}}\right)-2 \mu\left(\frac{\mathrm{z}_{\mathrm{U}}}{3}+\frac{1-\frac{\mathrm{r}_{0}}{\mathrm{R}}}{\mathrm{z}_{0}}-\frac{1-\frac{\mathrm{r}_{0}^{2}}{\mathrm{R}^{2}}}{\mathrm{Z}}\right)\right]
$$

where $e=V_{1} / V$ - coefficient of deceleration of the wind wheel, and $V_{1}=1 / 3 \cdot V-$ loss of wind speed in the plane of the wind wheel; $Z=\left(\omega_{-} \mathrm{R}\right) / \mathrm{V}$ - speed of the wind turbine, and $\omega \mathrm{W}$ - angular velocity of the wind wheel; $\mathrm{Z}_{0}$ - distance from the axis of the wind wheel to the beginning of the blade; $\mu=\mathrm{Cx} / \mathrm{Cy}$ is the reverse quality of the wind wheel blade, determined from the $C x$ and $C y$ diagrams for specific blade profiles; $Z_{U}=\omega R /\left(V-V_{1}\right)-$ the relative number of modules for the end of the blade [11].

Simulation of the drive and the generators is suggested using the developed and most frequently used mathematical models of EPS [10].

\section{Real Time Hybrid Simulation of Electrical Power Systems}

HRTSim is based on the concept of hybrid simulation and has the form of parallel, multiprocessor, hybrid software-hardware real time system combining adaptable complex of three-phase specialized hybrid processors (SHP) of all simulated power system elements and data management system (DMS) (Fig 2). 


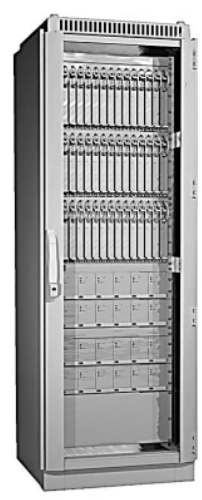

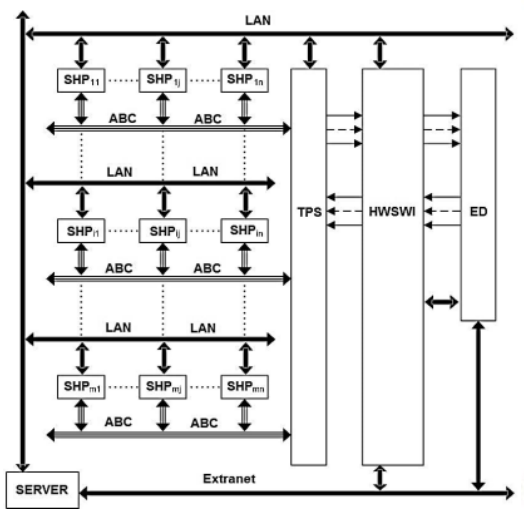

(a)

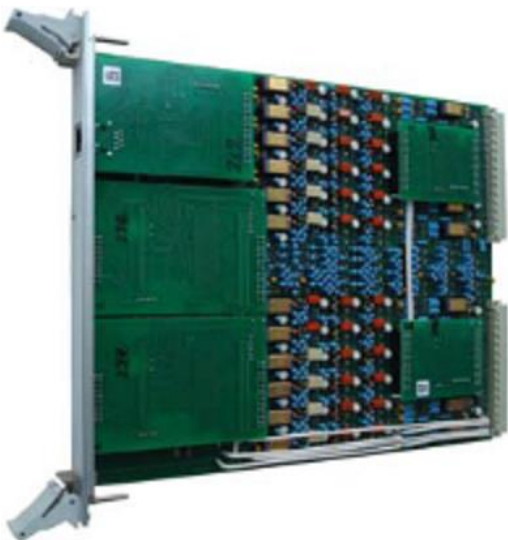

(b)

Fig 2. (a) HRTSim and its operational diagram: SHP - specialized hybrid processors; LAN - local area network; TPS - three-phase switch; HWSWI - hardware/software interface; ED - external device; ABC - three-phase input/output of SHP; (b) SHP of power line.

The hybrid simulation technology developed at the Tomsk Polytechnic University is analogue modeling, digital and physical. Each approach is used to solve individual subtasks, which solve the general problem of EPS modeling. The key task is to avoid a methodological error in solving differential equations [12-13]. The analogue part of the HRTSim provides continuous implicit integration, the digital part - provides flexibility, rapid and accurate control of model parameters and visualization of results; the existence of a physical layer allows us to unite the models of power system elements by converting mathematical values into physical voltages and currents of the model while minimizing the volume of digital information exchange [14].

\section{Conclusions}

In this paper, a detailed method for modeling a wind farm for analyzing the system stability studies and transient processes was proposed. In this model, individual wind turbines within the wind farm could receive different incoming winds. And also a new model of a wind turbine was proposed.

Also in this article we propose the use of HRTSim for detailed modeling of a large wind power plant, the use of which solves an important problem, given the number of wind turbines, various wind farm models and wind distribution changes. The calculations of mathematical models of the wind and wind turbine, even with detailed modeling, will have high-precision results, and the calculation speed will be rapid due to the features of the HRTSim of the EPS and its advantages in comparison with other Hardware and software simulation tools.

This work was supported by the Ministry of Education and Science of the Russian Federation under the governmental grant "Science" No. 13.5852.2017/БЧ (Development of the concept for comprehensive validation of calculating modes and processes in electric power system and tools of its realization).

\section{References}

1. GWEC. Global Wind Statistics, 2015.2.10, Brussels, Belgium (2014)

2. Hongzhi Liu, Zhe Chen, IEEE APPEEC 978-1-4577-0547-2/12/ (2012) 
3. R. Ufa, A. Vasilev, A. Gusev, A. Suvorov, IFOST, 113 (2016), DOI: 10.1109/IFOST.2016.7884204

4. M. Andreev, Y. Borovikov, N. Ruban, A Khlebov, MATEC Web Conf. 91, 01048, (2016), DOI:10.1051/matecconf/20179101048

5. A. Gusev, A. Suvorov, A. Sulaymanov, ICIEAM 127470, 7911415, (2016), DOI:10.1109/ICIEAM.2016.7911415

6. I. Tsoy., N. Kosmynina, IOP Conf. Series, 27 (1), 012065 (2015), DOI:10.1088/1755$1315 / 27 / 1 / 012065$

7. A. Askarov, M. Kondrashov, N.Kosmynina, MATEC Web Conf. 37, 01004 (2015), DOI: $10.1051 /$ matecconf $/ 20153701004$

8. S. Obukhov, I. Plotnikov, E. Sarsekeev, Mod. problems sci. educ. 5, 137 (2013)

9. IEC 61400-1 Third edition (2005)

10. D. Hansen, DIgSILENT technical documentation (2010)

11. N. Ruban, A. Gusev, A. Pishchulin, MATEC Web Conf. 37, 01046 (2015), DOI:10.1051/matecconf $/ 20153701046$

12. A. Suvorov, A. Gusev, A. Sulaymanov, O. Suslova, MATEC Web Conf. 91, 01052 (2017), DOI:10.1051/matecconf/20179101052

13. N. Ruban, M. Andreev, Y. Borovikov, ICIEAM 127470, 7910994, (2016), DOI:10.1109/ICIEAM.2016.7910994 